\title{
Adaptive remote radio head control for cloud radio access networks
}

\author{
Junsu Choi ${ }^{1}$, Illsoo Sohn² and Kwang Bok Lee ${ }^{1 *}$
}

\begin{abstract}
In this paper, we develop an adaptive remote radio head (RRH) control scheme to maximize the network capacity of frequency division duplexing (FDD)-based cloud radio access networks. We focus on a realistic performance metric that considers reference signal (RS) transmission overhead. Finding the optimal subset of RRHs that maximize the network capacity is formulated as an integer programming problem. We develop two efficient algorithms based on greedy search and linear programming relaxation combined with gradient ascent search, respectively. Our simulation results reveal that a larger number of antennas do not always guarantee capacity increase in real communication environments due to RS transmission overhead. The proposed scheme adaptively determines the subset of RRHs considering RS transmission overhead and provides significant capacity gain over previous approaches.
\end{abstract}

Keywords: Cloud radio access networks, Remote radio head control, Reference signal overhead

\section{Introduction}

The cloud radio access network (C-RAN) is recognized as one of the key enabling technologies for beyond $4 \mathrm{G}$ (B4G) or $5 \mathrm{G}$ wireless communications systems because of its ability to mitigate inter-cell interference and enhance the link quality [1-4]. Evolution to the C-RAN has already started in advanced commercial long-term evolution (LTE) networks [5-7]. The principal difference of a CRAN over a conventional cellular system is that individual management of cell operation becomes meaningless. In a C-RAN, a large number of remote radio heads (RRHs), i.e., groups of co-located antennas, are scattered over the network, and they are connected to and controlled by a central processor (CP) through fronthaul. Multiple RRHs are then involved in user service by transmitting cooperative signals. Figure 1 depicts the concept of a C-RAN as a single virtual network.

In a theoretical view, the network capacity increases as more RRHs and users are involved in cooperative transmission. However, realistic C-RANs have some practical constraints that limit RRH cooperation. Firstly, imperfect fronthaul imposes limitations on RRH cooperation. Cooperative transmission requires baseband signal

*Correspondence: klee@snu.ac.kr

1 Department of Electrical and Computer Engineering, INMC, Seoul National University, Gwanak-ro, 08826 Seoul, South Korea

Full list of author information is available at the end of the article exchange between the CP and RRHs, which is enabled by fronthaul links. Therefore, when fronthaul links have finite capacity, RRH cooperation is inevitably limited. In [8-13], RRHs are modeled as relays between the users and $\mathrm{CP}$, and compression/decompression strategies are developed considering the finite capacity of fronthaul links. In $[14,15]$, RRH clustering schemes are developed considering the capacity limit of fronthaul.

Considering frequency division duplexing (FDD)-based C-RANs, acquiring channel state information (CSI) at the $\mathrm{CP}$ requires downlink antenna training and uplink CSI feedback from users. Antenna training and CSI feedback consume precious time and frequency resources which increase with the number of RRHs involved in user service. Considering that the available time and frequency resources are limited, those overheads might be a bottleneck for RRH cooperation in an FDD-based C-RAN where a large number of RRHs are deployed for user service. In $[16,17]$, RRH cooperation strategies are developed to reduce the overhead required for acquiring CSI at the CP.

Here, we introduce a completely new approach to maximize the network capacity of an FDD-based C-RAN. We focus on the trade-off between the antenna processing gain and reference signal (RS) overhead. In a practical FDD-based C-RAN, antennas transmit RSs for acquiring CSI at the CP. Upon receipt of the RSs, users estimate their

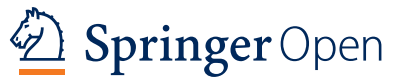

(c) 2016 The Author(s). Open Access This article is distributed under the terms of the Creative Commons Attribution 4.0 International License (http://creativecommons.org/licenses/by/4.0/), which permits unrestricted use, distribution, and reproduction in any medium, provided you give appropriate credit to the original author(s) and the source, provide a link to the Creative Commons license, and indicate if changes were made. 


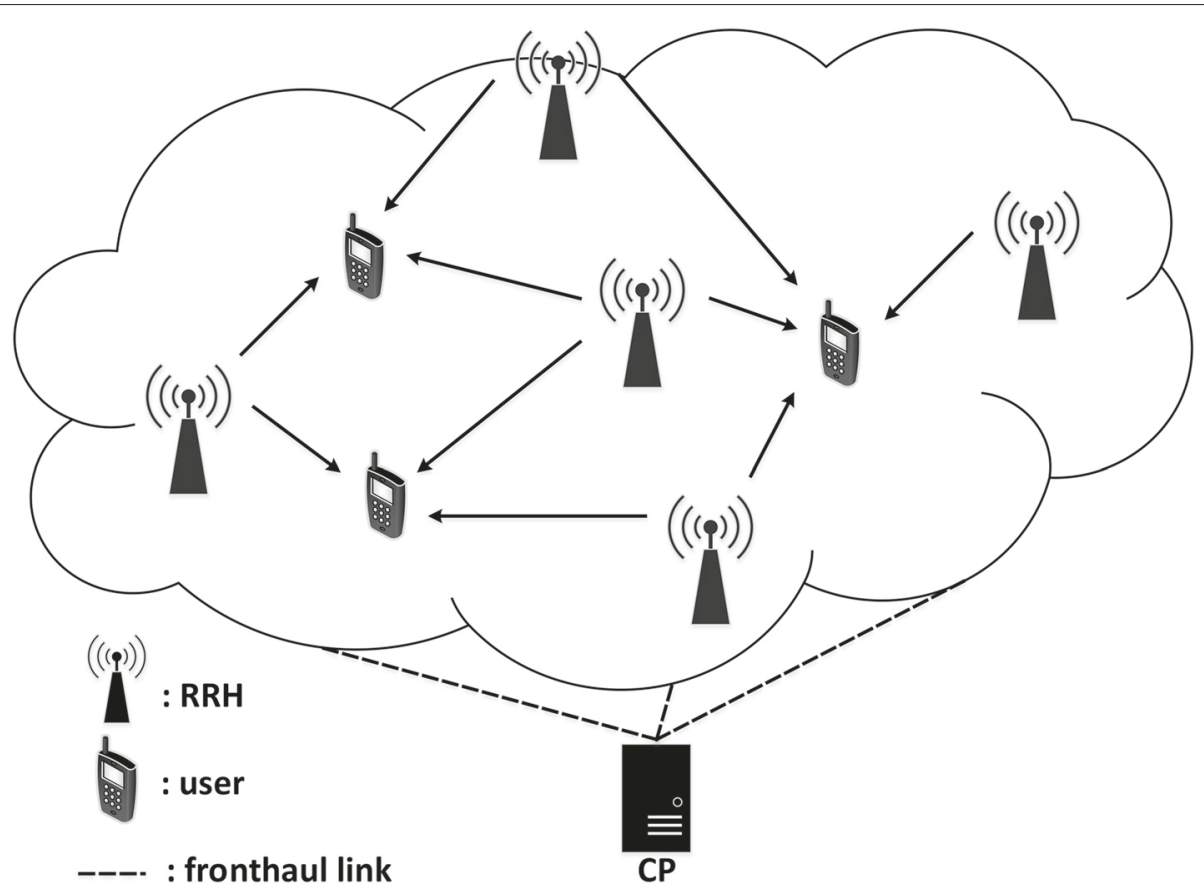

Fig. 1 Cloud radio access network

channels and feed back the CSI to the CP. In $[18,19]$, it is shown that RSs of different antennas should be conveyed on mutually orthogonal time and frequency resources for accurate channel estimation, which leads to RS overhead linearly increasing with the number of antennas. This has been a principal design guideline for recent OFDM-based wireless systems. For example, in commercial LTE networks [20], the CSI-RS overhead in a subframe grows from 1.19 to $4.76 \%$ as the number of antennas increases from 2 to 8 .

As more antennas participate in cooperative transmission, the antenna processing gain increases while RS transmission consumes a larger fraction of time and frequency resources, which otherwise would be dedicated for data transmission. Considering the trade-off relationship, we need to investigate the impact of RS transmission overhead on the network capacity of an FDD-based CRAN in which a large number of antennas are scattered for user service. We then also need to find the best subset of RRHs that maximize the network capacity considering both the antenna processing gain and RS transmission overhead.

In this paper, we investigate the impact of RSs on the network capacity of an FDD-based C-RAN with comprehensive consideration of the antenna processing gain and RS transmission overhead. To this end, we consider a realistic performance metric that takes into account both the antenna processing gain and RS transmission overhead. Using the metric, we formulate an integer programming
(IP) problem that determines the best RRH subset leading to maximum network capacity. To the best of our knowledge, the trade-off between the antenna processing gain and RS overhead has never been comprehensively considered for network capacity maximization in the literature of C-RAN control.

Solving an IP problem normally requires a computationally demanding task. To reduce the computational burden, we also develop two efficient algorithms based on greedy search and linear programming (LP) relaxation combined with gradient ascent search, respectively. With the greedy search-based algorithm, the computational complexity increases with the square of the network size. The LP relaxation-based algorithm leads to further reduction of the computational burden for an extremely large-scale network, i.e., linear increase of computational complexity with the network size at the expense of performance loss compared to the greedy search-based one.

The rest of this article is organized as follows. Section 2 describes the system model. In Section 3, we formulate the RRH subset selection problem and develop an efficient algorithm to reduce the computational burden. We present simulation results in Section 4, and concluding remarks are given in Section 5.

Notations: Lowercase and uppercase boldface letters denote vectors and matrices, respectively. $\mathbf{I}_{N}$ represents the $N \times N$ identity matrix. The superscripts " $\mathrm{T}$ " and "*" denote transpose and conjugate transpose operators, respectively. Lastly, we will use $\mathbb{E}[\cdot]$ for expectation. 


\section{System model}

We consider an FDD-based C-RAN in which there are $L$ RRHs and $K$ users. We assume ideal fronthaul links with zero latency. The $l$ th RRH is equipped with $m_{l}$ antennas such that $M=\sum_{l=1}^{L} m_{l}$, and the $k$ th user is equipped with $n_{k}$ antennas such that $N=\sum_{k=1}^{K} n_{k}$. The channel from the $l$ th RRH to the $k$ th user is denoted as $\mathbf{H}_{k, l} \in \mathbb{C}^{n_{k} \times m_{l}}$. Therefore, the aggregate channel for the $k$ th user, $\mathbf{H}_{k} \in$ $\mathbb{C}^{n_{k} \times M}$, is presented by $\mathbf{H}_{k}=\left[\mathbf{H}_{k, 1} \mathbf{H}_{k, 2} \cdots \mathbf{H}_{k, L}\right]$, and the aggregate channel for all the users, $\mathbf{H} \in \mathbb{C}^{N \times M}$, is given by

$$
\mathbf{H}=\left[\mathbf{H}_{1}^{\mathrm{T}} \mathbf{H}_{2}^{\mathrm{T}} \cdots \mathbf{H}_{K}^{\mathrm{T}}\right]^{\mathrm{T}} .
$$

The path loss from the $l$ th $R R H$ to the $k$ th user is denoted as $\beta_{k, l}$, and hence, if we assume rich scattering, it holds that

$$
\mathbb{E}\left[\mathbf{H}_{k, l} \mathbf{H}_{k, l}^{*}\right]=m_{l} \beta_{k, l} \mathbf{I}_{n_{k}},
$$

Let us denote the total amount of time and frequency resources available for both data and RSs of antennas during the coherence interval as $U$. Let us also assume that RSs of different antennas are transmitted using mutually orthogonal time and frequency resources and denote the amount of resources dedicated for RS transmission of an antenna as $\alpha$. The total amount of resources dedicated for RSs then linearly increases with the number of antennas involved in cooperative transmission, which ends up with the fraction of the remaining resources available for data transmission presented by $\frac{U-\alpha M}{U}$. Accordingly, considering RS transmission overhead, perfect CSI at the CP leads to the effective network capacity described by

$$
C=\left(\frac{U-\alpha M}{U}\right) \log _{2} \operatorname{det}\left(\mathbf{I}_{N}+\frac{P}{\sigma^{2}} \mathbf{H H}^{*}\right),
$$

where $P$ is the transmission power per spatial stream and $\sigma^{2}$ is the noise power. Note that (3) comprehensively considers both the RS overhead and antenna processing gains including spatial multiplexing and diversity gains. We use (3) as the performance metric for the FDD-based C-RAN.

\section{Adaptive RRH control}

\subsection{Problem formulation}

We introduce a diagonal RRH selection matrix $\mathbf{S} \in \mathbb{C}^{M \times M}$ which is given by

$$
\mathbf{S}=\left[\begin{array}{cccc}
\mathbf{S}_{1} & \mathbf{0} & \ldots & \mathbf{0} \\
\mathbf{0} & \mathbf{S}_{2} & \ldots & \mathbf{0} \\
\vdots & \vdots & \ddots & \vdots \\
\mathbf{0} & \mathbf{0} & \ldots & \mathbf{S}_{L}
\end{array}\right]
$$

where $\mathbf{S}_{l} \in \mathbb{C}^{m_{l} \times m_{l}}$ is also a diagonal matrix which is given by $\mathbf{S}_{l}=\operatorname{diag}\left(s_{l}, s_{l}, \ldots, s_{l}\right)$, where $s_{l}$ is a binary integer variable whose value is either 1 if the $l$ th $R R H$ is selected or 0 otherwise. Then, with the selected subset of
RRHs, the number of antennas that transmit RSs is given by $\sum_{l=1}^{L} s_{l} m_{l}$, and hence, the fraction of time and frequency resources available for data transmission becomes $\frac{U-\alpha \sum_{l=1}^{L} s_{l} m_{l}}{U}$. Therefore, the network capacity is given by

$$
\begin{aligned}
C & =\left(\frac{U-\alpha \sum_{l=1}^{L} s_{l} m_{l}}{U}\right) \log _{2} \operatorname{det}\left(\mathbf{I}_{N}+\frac{P}{\sigma^{2}} \mathbf{H S}(\mathbf{H S})^{*}\right) \\
& =\left(\frac{U-\alpha \sum_{l=1}^{L} s_{l} m_{l}}{U}\right) \log _{2} \operatorname{det}\left(\mathbf{I}_{N}+\frac{P}{\sigma^{2}} \mathbf{H S H}^{*}\right),
\end{aligned}
$$

where the second equality is derived from $\mathbf{S S}^{*}=\mathbf{S}$. Note that the CP obtains instantaneous CSI only after RS transmission of all antennas and CSI feedback from users, which means that the $\mathrm{CP}$ does not have instantaneous CSI of all antennas at the stage of RRH subset selection. Instead, we assume that the $\mathrm{CP}$ has second-order statistics information of CSI based on the long-term observation of channels, i.e., long-term path loss from the $l$ th RRH to the $k$ th user, where $l=1,2, \ldots, L$ and $k=1,2, \ldots, K$.

We take the expectation of (5) over the short-term small-scale fading, and then, the long-term ergodic network capacity is given by

$$
\mathbb{E}[C]=\left(\frac{U-\alpha \sum_{l=1}^{L} s_{l} m_{l}}{U}\right) \mathbb{E}\left[\log _{2} \operatorname{det}\left(\mathbf{I}_{N}+\frac{P}{\sigma^{2}} \mathbf{H S H}^{*}\right)\right] .
$$

Applying Jensen's inequality, ${ }^{1}$ the ergodic network capacity is approximately given by

$\bar{C} \approx\left(\frac{U-\alpha \sum_{l=1}^{L} s_{l} m_{l}}{U}\right) \log _{2} \operatorname{det}\left(\mathbf{I}_{N}+\frac{P}{\sigma^{2}} \mathbb{E}\left[\mathbf{H S H}^{*}\right]\right)$.

In addition, $\mathbb{E}\left[\mathbf{H}_{k_{1}, l} \mathbf{H}_{k_{2}, l}^{*}\right]=\mathbf{0}$ for $k_{1} \neq k_{2}$, $l=1,2, \ldots, L$ due to independent fading. Therefore, $\mathbb{E}\left[\right.$ HSH $\left.^{*}\right]$ becomes an $N \times N$ diagonal matrix which is given by

$$
\mathbb{E}\left[\mathbf{H S H}^{*}\right]=\left[\begin{array}{cccc}
\mathbb{E}\left[\mathbf{H}_{1} \mathbf{S} \mathbf{H}_{1}^{*}\right] & \mathbf{0} & \ldots & \mathbf{0} \\
\mathbf{0} & \mathbb{E}\left[\mathbf{H}_{2} \mathbf{S H}_{2}^{*}\right] & \ldots & \mathbf{0} \\
\vdots & \vdots & \ddots & \vdots \\
\mathbf{0} & \mathbf{0} & \ldots & \mathbb{E}\left[\mathbf{H}_{K} \mathbf{S H}_{K}^{*}\right]
\end{array}\right]
$$

where $\mathbb{E}\left[\mathbf{H}_{k} \mathbf{S H}_{k}^{*}\right]=\left(\sum_{l=1}^{L} s_{l} m_{l} \beta_{k, l}\right) \mathbf{I}_{n_{k}}$ from (2) for $k=$ $1,2, \ldots, L$. Accordingly, $\mathbf{I}_{N}+\frac{P}{\sigma^{2}} \mathbb{E}\left[\mathbf{H S H} \mathbf{H}^{*}\right]$ is also a diagonal matrix, and its determinant is the product of its diagonal elements. From the above analysis, it holds that 


$$
\operatorname{det}\left(\mathbf{I}_{N}+\frac{P}{\sigma^{2}} \mathbb{E}\left[\mathbf{H S H}^{*}\right]\right)=\prod_{k=1}^{K}\left(1+\frac{P}{\sigma^{2}} \sum_{l=1}^{L} s_{l} m_{l} \beta_{k, l}\right)^{n_{k}} .
$$

Substituting (9) for the determinant term in (7) gives

$$
\bar{C}=\left(\frac{U-\alpha \sum_{l=1}^{L} m_{l} s_{l}}{U}\right) \sum_{k=1}^{K} n_{k} \log _{2}\left(1+\frac{P}{\sigma^{2}} \sum_{l=1}^{L} s_{l} m_{l} \beta_{k, l}\right) .
$$

Accordingly, we finally formulate the RRH subset selection problem as

$$
\begin{array}{ll}
\text { maximize } & \bar{C} \\
\text { subject to } & s_{l} \in\{0,1\}, l=1,2, \ldots, L .
\end{array}
$$

\subsection{Analysis on the optimal RRH subset}

In this subsection, we discuss the existence of the optimal RRH subset as the solution of (11) and the impact of the network environment on the optimal RRH subset. Let us assume that a very small number of RRHs are involved in cooperative transmission, i.e., the capacity reduction from RS overhead is negligible. Then, $\frac{\alpha}{U} \sum_{l=1}^{L} m_{l} s_{l} \approx$ 0 , and $\bar{C} \approx \sum_{k=1}^{K} n_{k} \log _{2}\left(1+\frac{P}{\sigma^{2}} \sum_{l=1}^{L} s_{l} m_{l} \beta_{k, l}\right)$, which increases with the antenna processing gain as more RRHs are involved in cooperative transmission. On the other hand, let us assume that a very large number of RRHs are involved in cooperative transmission, i.e., the log terms in $\bar{C}$ reach the saturation region. Then, involving more RRHs in cooperative transmission leads to negligible increase of those log terms while the $\left(\frac{U-\alpha \sum_{l=1}^{L} m_{l} s_{l}}{U}\right)$ term leads to linear capacity reduction with the number of antennas involved in cooperative transmission.

The above reasoning implies that $\bar{C}$ is maximized with the best subset of RRHs balancing the antenna processing gain and RS overhead, i.e., finding the optimal solution of (11) leads to network capacity maximization in consideration of the antenna processing gain and RS overhead. If we increase the transmission power per spatial stream, $P$ in (10), the log terms in (10) reaches the saturation region more quickly with selection of more RRHs. Therefore, as the network allocates more power for user services, the optimal RRH subset of (11) consists of a smaller number of RRHs, which leads to RS transmission consuming a smaller potion of time and frequency resources.

\subsection{Proposed algorithm}

The binary integer variables $s_{1}, s_{2}, \ldots, s_{L}$ lead to the RRH subset selection problem in (11) belonging to integer programming (IP). There exists no general solution for IP, and the computational complexity for finding the optimal solution exponentially increases in the order of $2^{L}$, which

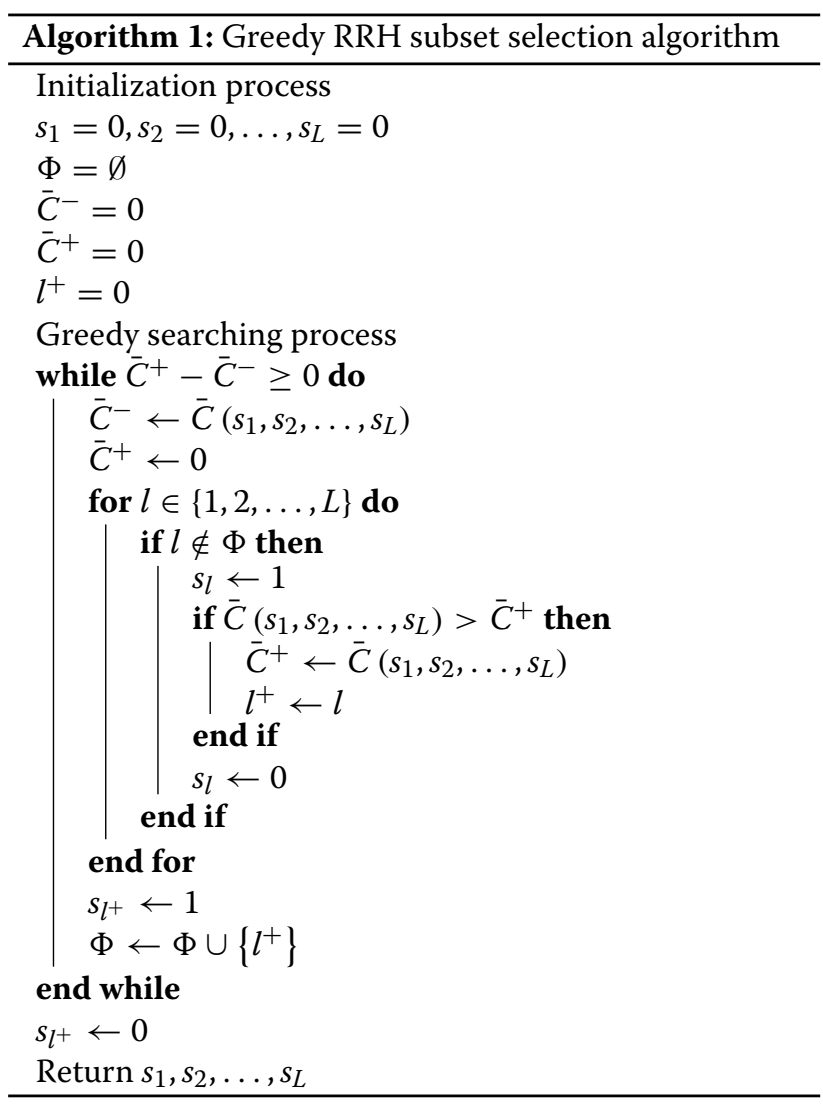

would be a substantial burden as the network size grows. Hence, we develop practical algorithms that efficiently find approximate solutions of the RRH subset selection problem in (11). The first algorithm is a greedy searchbased algorithm described in Algorithm 1. In the first searching process, the set of selected RRHs, i.e, $\Phi$ is set to $\emptyset$. The greedy search algorithm then compares the value of $\bar{C}$ with sequentially selecting RRHs not in $\Phi$ and augments $\Phi$ by selecting the RRH leading to maximum capacity increase. Greedy searching processes continue until capacity loss from RS overhead dominates capacity increase from the antenna processing gain, i.e., additional augment of $\Phi$ results in decrease of $\bar{C}$. The greedy search algorithm then returns the final result $s_{1}, s_{2}, \ldots, s_{L}$.

In the $j$ th searching process, the greedy search algorithm goes through $L-j+1$ cases to find the next member of $\Phi$, which leads to computational complexity increasing on the order of $O\left(L^{2}\right)$. Considering that the proposed algorithm is periodically performed with long-term intervals, the computational complexity of $O\left(L^{2}\right)$ is assessed to be affordable for the CP. However, in an extremely large-scale network, it might be a severe burden, and we develop another efficient algorithm that finds an approximate solution of (11) with further reduced computational complexity. 
The second algorithm makes use of the linear programming relaxation [21] and gradient ascent search [22] as described in Algorithm 2. We firstly relax the binary integer constraints on variables $s_{1}, s_{2}, \ldots, s_{L}$ to real number constraints with

$$
0 \leq s_{l} \leq 1, \quad l=1,2, \ldots, L
$$

Then, we initialize the aggregated vector of the variables $\mathbf{x}=\left[s_{1}, s_{2}, \ldots, s_{L}\right]$ to be $\mathbf{x}_{0}$. After initialization, $\mathbf{x}$ is iteratively updated as $\mathbf{x}_{t}$ from $\mathbf{x}_{t-1}$ for $t=1,2,3 \ldots$ according to the update rule shown below.

$$
\mathbf{x}_{t}=f\left(\mathbf{x}_{t-1}+\delta \nabla \bar{C}\left(\mathbf{x}_{t-1}\right)\right), \quad t=1,2,3, \ldots
$$

where $\nabla \bar{C}\left(\mathbf{x}_{t-1}\right)$ is the gradient vector of $\bar{C}$ with respect to $\mathbf{x}$ at $\mathbf{x}_{t-1}, \delta$ is the step size, and $f(\mathbf{x})$ is the projection of vector $\mathbf{x}$ to the nearest vector $\tilde{\mathbf{x}}$ which satisfies the constraints $0 \leq s_{l} \leq 1$ for $l=1,2, \ldots, L$, i.e., the projection is described by

$$
s_{l} \leftarrow\left\{\begin{array}{ll}
1, & s_{l}>1, \\
0, & s_{l}<0, \\
s_{l}, & \text { otherwise, }
\end{array} \quad l=1,2, \ldots, L\right.
$$

The iterative update continues until the difference $\left\|\mathbf{x}_{t}-\mathbf{x}_{t-1}\right\|$ becomes smaller than a threshold $\eta$, where $\|\mathbf{x}\|$ is the norm of of vector $\mathbf{x}$. Once the update process terminates, we denote the index set for the $r\left(\sum_{l=1}^{L} s_{l}\right)$ largest elements of $\mathbf{x}_{t}$ as $\Phi$, where $r(g)$ is the function which returns the nearest integer from scalar $g$. Then,

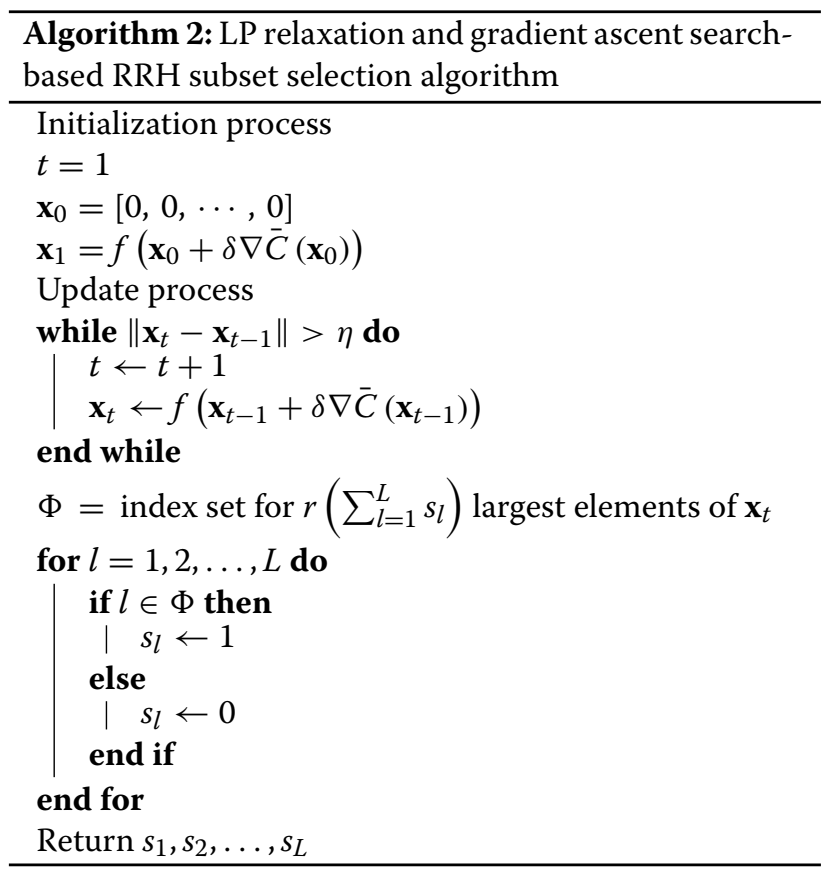

variables $s_{1}, s_{2}, \ldots, s_{L}$ are rounded up to satisfy the original binary integer constraint as

$$
s_{l} \leftarrow\left\{\begin{array}{l}
1, l \in \Phi \\
0, \text { otherwise, } \quad l=1,2, \ldots, L .
\end{array}\right.
$$

Lastly, the algorithm returns the final result $s_{1}, s_{2}, \ldots, s_{L}$. Extensive simulations reveal that the choice of initial point $\mathbf{x}_{0}$ has negligible impact on the final results, and hence, we simply set $\mathbf{x}_{0}=[0,0, \cdots, 0]$ in the algorithm.

At each iteration of the proposed algorithm, the computational complexity for gradient calculation is proportional to the number of RRHs $L$. However, the number of total iterations does not increase with growth of $L$, and it rather reaches saturation once $L$ becomes large enough as we will validate later in Section 4 . Therefore, the overall computational complexity is approximated as $O(L)$, which would be affordable for the CP even in an extremely large-scale network.

\section{Simulation results}

We set a C-RAN, where users and RRHs are distributed within a service area of $1000 \times 1000 \mathrm{~m}$, where the path loss from an RRH to a user is determined by the distance with path loss exponent 4 , and the noise power $\sigma^{2}$ is $-100 \mathrm{dBm}$. The number of antennas at an RRH is randomly selected in the range of $[10,20]$, and each user is equipped with one antenna. We set the fraction of time and frequency resources dedicated for RS transmission per antenna to be $0.12 \%$, i.e., $\frac{\alpha}{U}=0.0012 .^{2}$ When we apply the proposed RRH control algorithms developed in Section 3.3, the step size $\delta$ and the threshold $\eta$ are set to 0.01 and 0.1 , respectively. Simulation parameters are summarized in Table 1, and other unspecified parameters will be specified at each simulation scenario.

We compare the performance of the proposed $\mathrm{RRH}$ control with other approaches: (1) the $L_{\text {near nearest } \mathrm{RRH}}$ selection approach introduced in [23], where each user selects the $L_{\text {near }}$ nearest RRHs considering the received signal strength and all the selected RRHs cooperatively serve the users; (2) the nearest and $\mathrm{SNR}_{\text {near }}$ range $\mathrm{RRH}$ selection approach introduced in [24], where each user

Table 1 Simulation parameters

\begin{tabular}{ll}
\hline Service area & $1000 \times 1000 \mathrm{~m}$ \\
Path loss exponent & 4 \\
Noise power $\sigma^{2}$ & $-100 \mathrm{dBm}$ \\
Number of antennas at an RRH & Randomly chosen \\
& from 10 to 20 \\
Fraction of resources for RS & $0.12 \%$ \\
transmission per antenna & \\
Gradient ascent step size $\delta$ & 0.01 \\
Gradient ascent threshold $\eta$ & 0.1 \\
\hline
\end{tabular}


selects the nearest RRH by default and also selects other RRHs from which the received signal strength is within the $\mathrm{SNR}_{\text {near }}$ range from the maximum received signal strength; and (3) no RRH control, where all RRHs are involved in cooperative transmission.

Figure 2 shows the number of selected RRHs versus the number of RRHs, $L$ when the number of users $K=$ 40 and the transmission power per spatial stream $P=$ $1 \mathrm{~mW}(0 \mathrm{dBm})$. Without RRH control, the number of selected RRHs inherently increases proportionally to $L$. Under both the nearest and $\mathrm{SNR}_{\text {near }}$ range $\mathrm{RRH}$ selection and $L_{\text {near }}$ nearest RRH selection, the number of selected RRHs also increases with growth of $L$ because increase of $L$ makes it more probable that different users select different sets of RRHs. On the other hand, the proposed RRH control selects a subset of RRHs to maximize network capacity in consideration of RS overhead according to (11). Therefore, the proposed RRH control with both the greedy search-based and LP relaxation-based algorithms (Algorithms 1 and 2 developed in Section 3.3) selects far smaller number of RRHs than the compared RRH control approaches, which leads to considerable performance improvement as will be discussed in the following.

Figure 3 shows network capacity versus the number of RRHs, $L$ in the previous simulation scenario. If RS overhead is neglected as done in the previous studies, network capacity would increase as more RRHs serve users. However, in reality, RS overhead severely degrades the network capacity without RRH control considering RS overhead. As shown in Fig. 3, network capacity begins to decrease with growth of $L$ after a point under all the compared RRH control approaches which do not consider RS overhead. On the other hand, the proposed RRH control leads to constant increase of network capacity with growth of $L$. Although one nearest RRH selection shows the least capacity degradation compared to the other compared approaches, it still wastes a larger fraction of resources for RS transmission than the proposed RRH control. The proposed RRH control with the greedy search-based and LP relaxation-based algorithms improves network capacity by 30 and $14 \%$, respectively, over one nearest RRH selection when $L=50$. As will be validated in the following, the LP relaxation-based algorithm reduces computational complexity compared to the greedy search-based algorithm. Although the LP relaxation-based algorithm shows performance degradation compared to the greedy searchbased algorithm, it still achieves better performance than the RRH control approaches which do not consider RS overhead.

Figure 4 shows the average number of required iterations for the LP relaxation-based RRH control algorithm in the above simulation scenario. When the number of RRHs $L$ is small, the average number of iterations increases with growth of $L$. However, it reaches saturation after $L=40$. As per our discussion in Section 3.3, gradient calculation in each iteration requires computational complexity of $O(L)$, and hence, the overall computational complexity could be also approximated as $O(L)$, which is far lighter than that of the greedy search-based RRH control algorithm, i.e., $O\left(L^{2}\right)$. Considering that RRH control is periodically performed with long-term intervals, this is assessed to be affordable for practical CPs even in extremely large-scale networks.

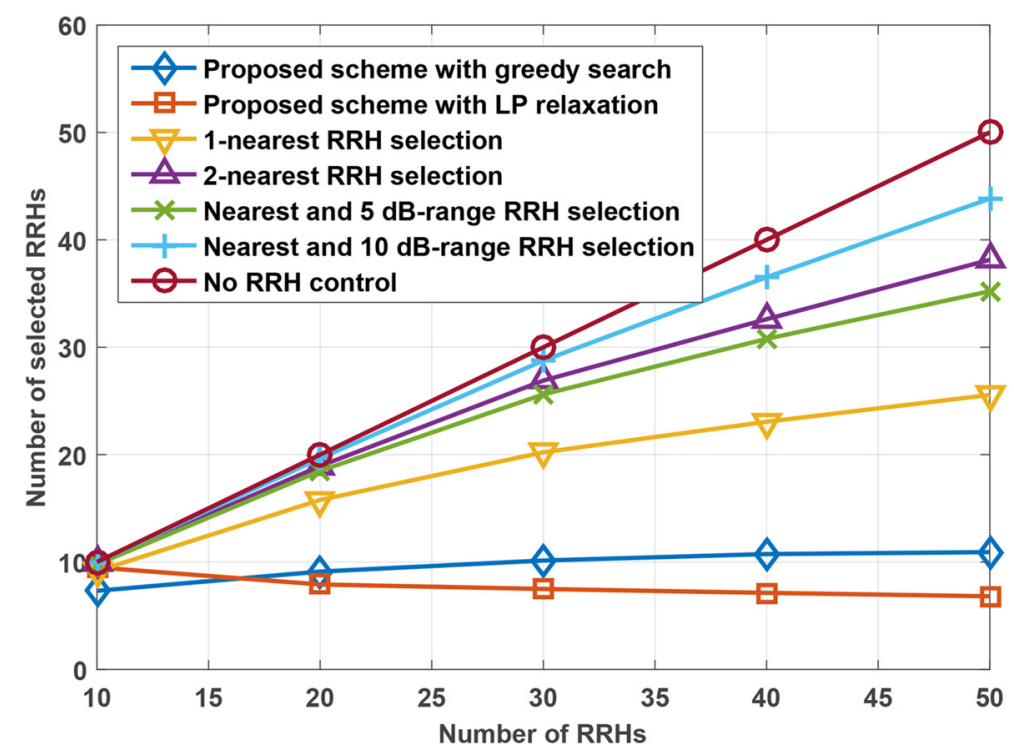

Fig. 2 The number of selected RRHs versus the number of RRHs 


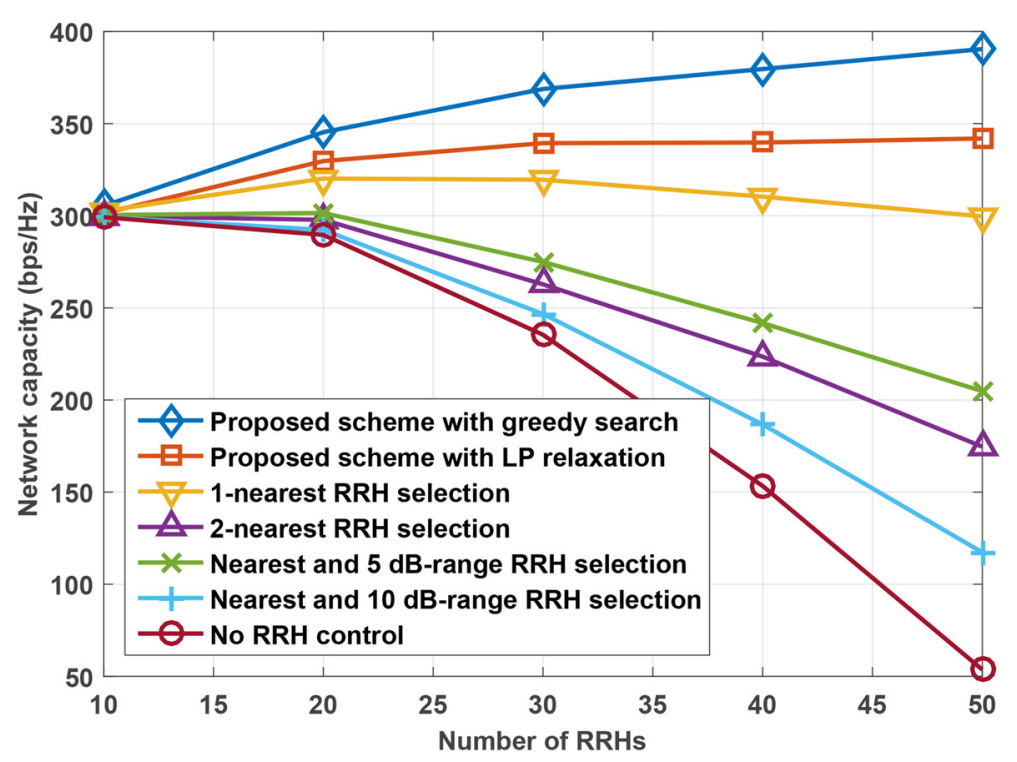

Fig. 3 Network capacity versus the number of RRHs

Figure 5 shows the comparison of the actual long-term ergodic capacity in the above simulation scenario with the approximation given by (7). As is well known, Jensen's inequality provides an upper bound for the expectation of the $\log$ function. Generally, the gap between the real value and the approximated value using Jensen's inequality becomes larger in the concave region of the function while it becomes smaller in the flat region of the function. In a C-RAN, users receive cooperate signals from RRHs near them, i.e., the network operates with a high received SNR.
It results in the network operating in the linear-like region of the $\log$ function, and the upper bound (7) provides very tight approximation for the actual ergodic network capacity as shown in the figure. The average normalized estimation error with both the greedy search-based and LP relaxation-based algorithms is less than $1 \%$.

Figure 6 shows network capacity versus the number of users, $K$ when the number of RRHs $L=40$ and transmission power per spatial stream $P=1 \mathrm{~mW}(0 \mathrm{dBm})$. Network capacity increases with growth of $K$ due to the

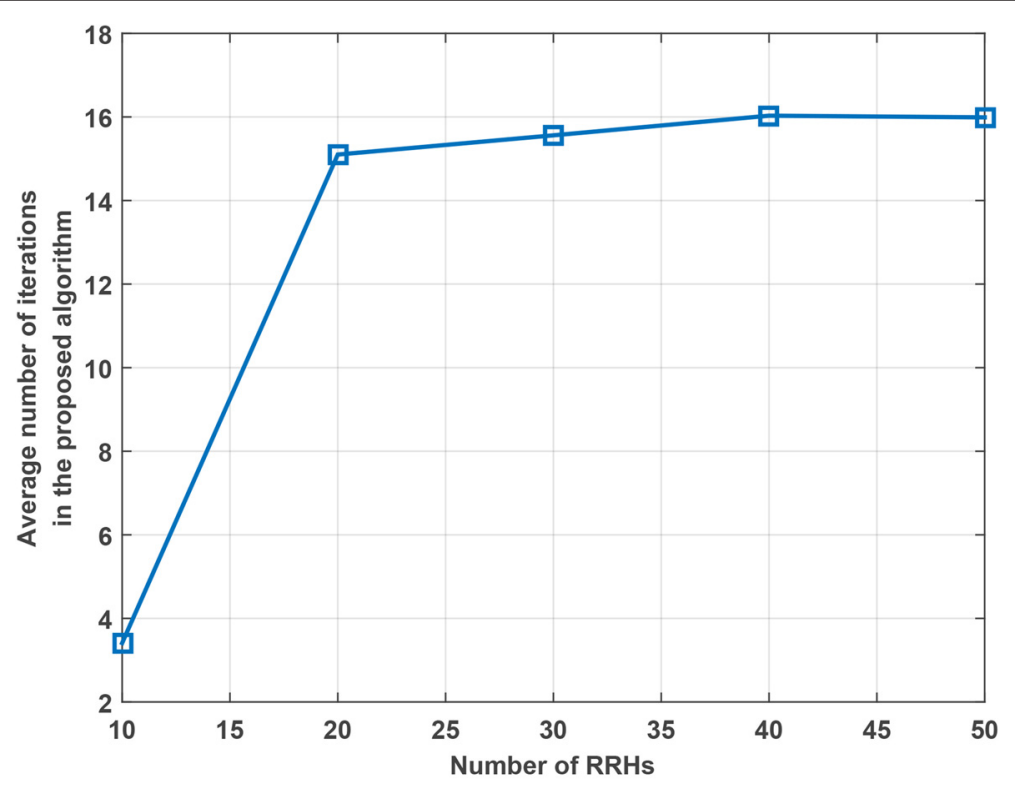

Fig. 4 The average number of iterations in Algorithm 1 


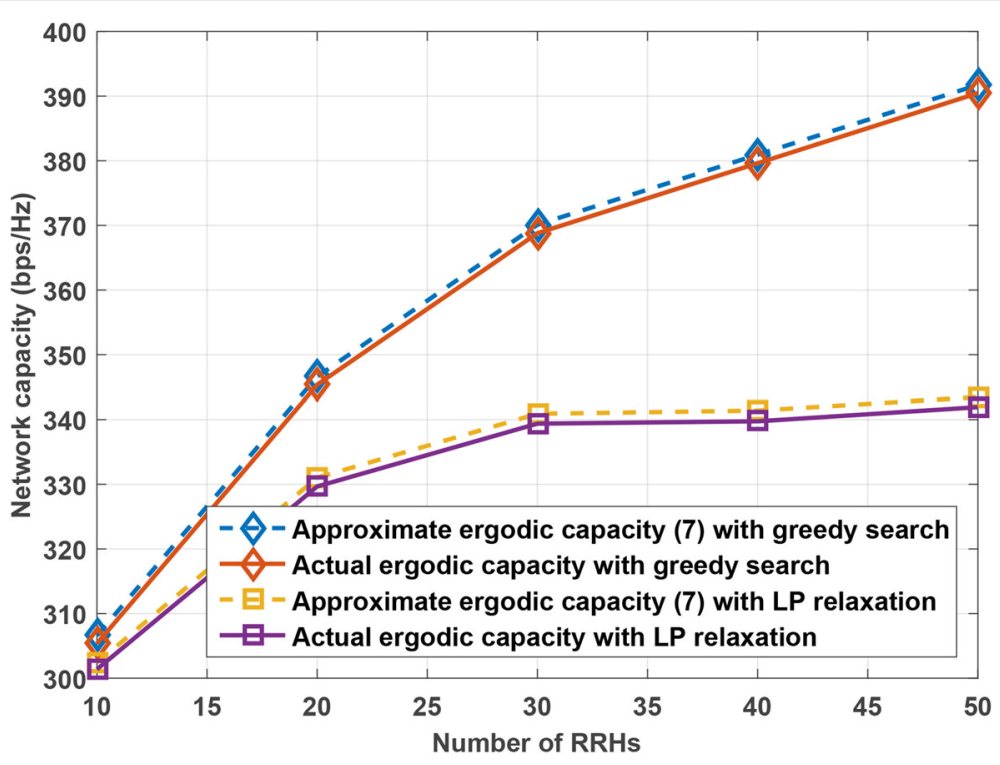

Fig. 5 Tightness of ergodic capacity approximation in the proposed scheme

increased spatial multiplexing gain. However, the RRH selection mechanisms of both the nearest and $\mathrm{SNR}_{\text {near }}$ range RRH selection and $L_{\text {nearest }}$ nearest RRH selection inherently increase the number of selected RRHs with growth of $K$. It results in a larger fraction of resources wasted by RS transmission with increase of $K$. On the other hand, the proposed RRH control selects a subset of RRHs in consideration of RS overhead, achieving considerable performance improvement over the existing schemes. When $K=60$, the proposed RRH control with the greedy search-based and LP relaxation-based algorithms achieves the capacity gains of 38 and $21 \%$, respectively, over one nearest RRH selection.

Figure 7 shows the number of selected RRHs versus transmission power per spatial stream, $P$ when the number of RRHs $L=40$ and the number of users $K=40$. Without RRH control, the number of selected RRHs remains the same as 40 . Likewise, the selection mechanism in the comparing schemes is not affected by transmission power, which also results in the number of selected RRHs being constant regardless of transmission power. On the other hand, the proposed RRH

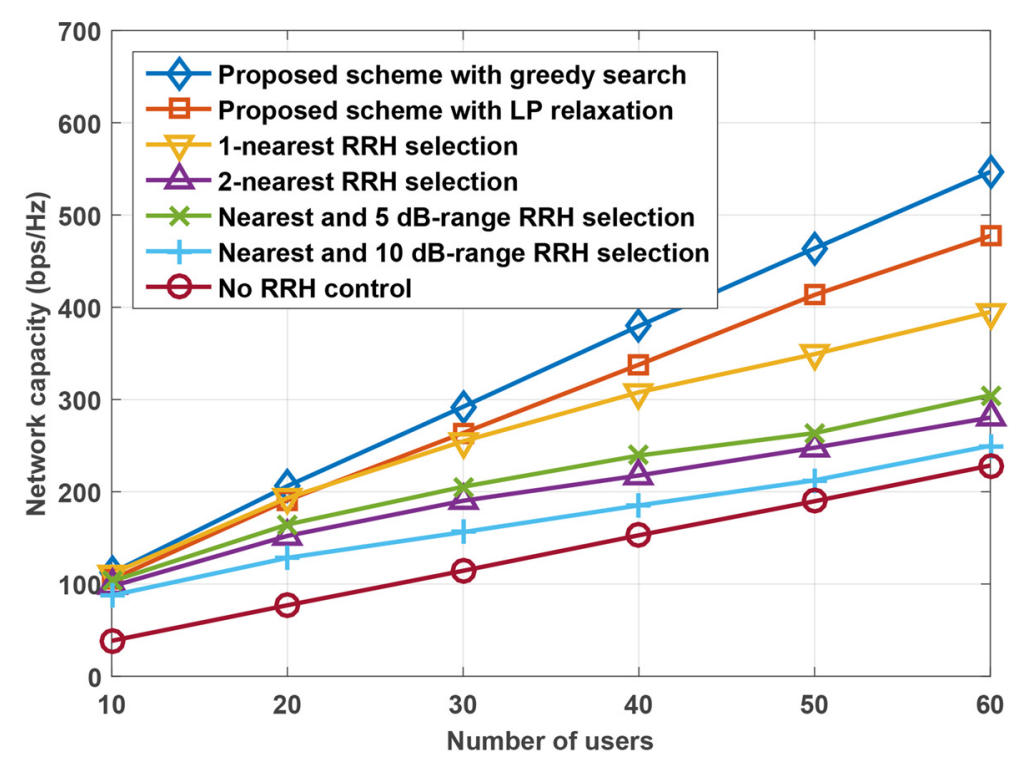

Fig. 6 Network capacity versus the number of users 


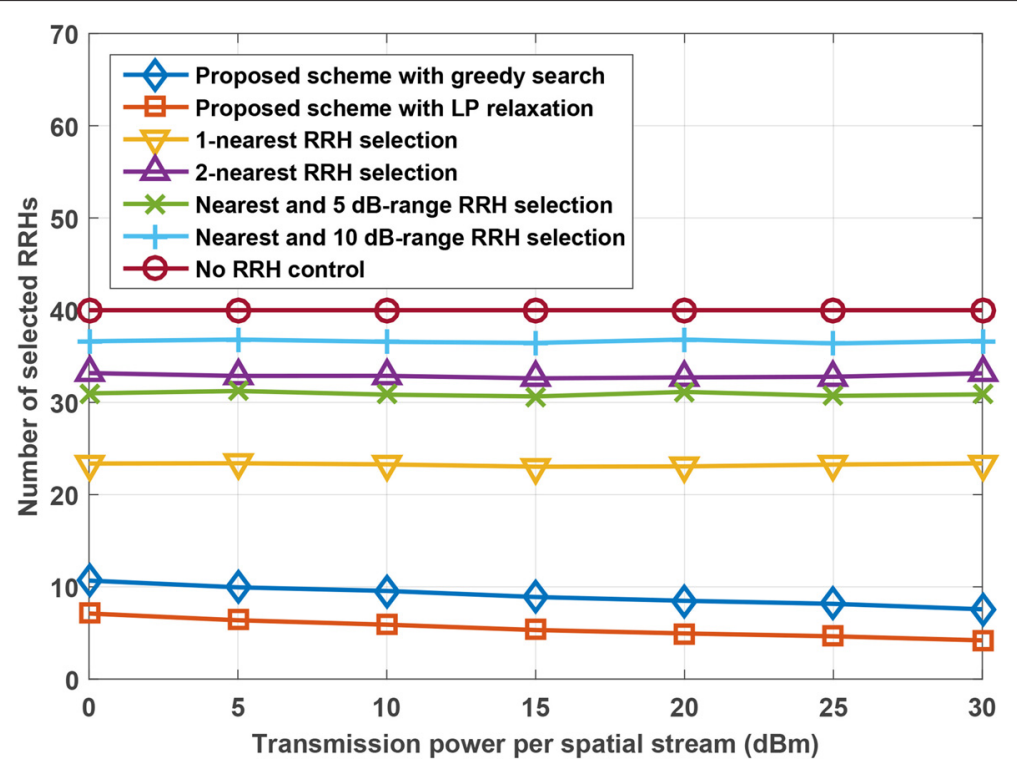

Fig. 7 The number of selected RRHs versus transmission power per spatial stream

control finds an RRH subset which balances between the antenna processing gain and RS overhead according to (11). When the received SNR is small, more antennas improve network capacity because the antenna processing gain is larger than the capacity loss from increased RS overhead. On the other hand, when the received SNR is sufficiently large, more antennas rather result in capacity loss from increased RS overhead due to the saturation characteristic of the log function. Therefore, the number of selected RRHs constantly reduces according to the increase of transmission power. For example, it reduces to about 8 and 4 , respectively, under the greedy search-based and LP relaxation-based algorithms when transmission power per spatial stream becomes $30 \mathrm{dBm}$.

Figure 8 shows network capacity versus transmission power per spatial stream, $P$ in the previous simulation scenario. Under all the comparing schemes, network capacity increases according to the growth of $P$. However, they do not consider RS overhead in RRH selection and waste a large fraction of time and frequency resources, which results in considerable performance degradation

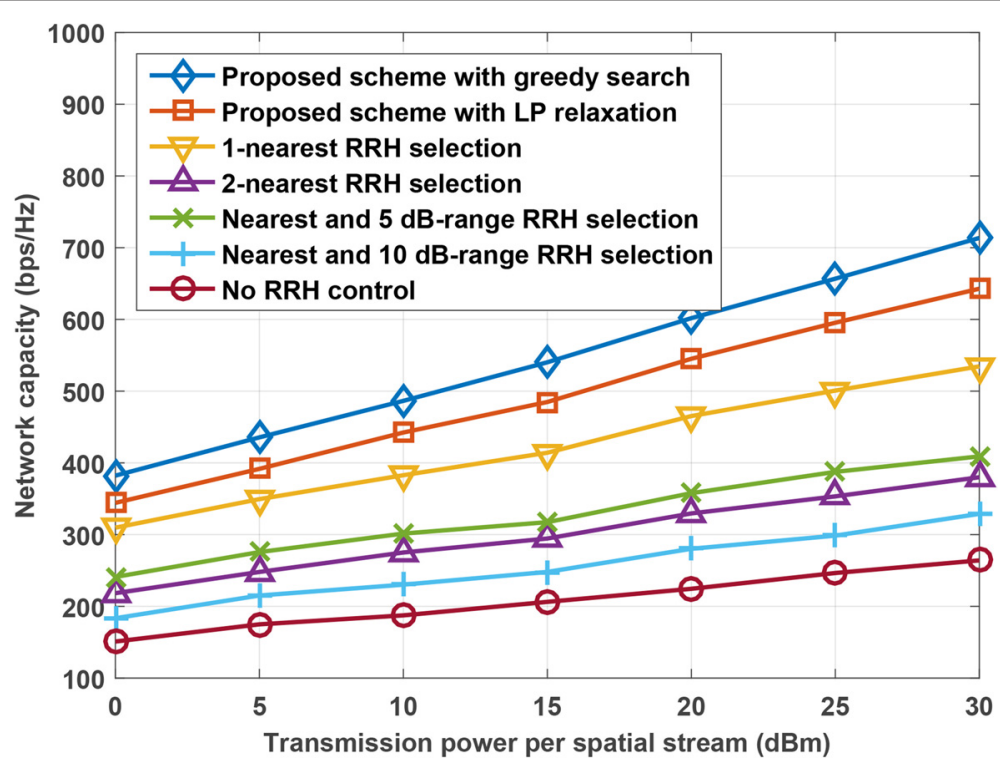

Fig. 8 Network capacity versus transmission power per spatial stream 
compared to the proposed RRH control. As per our discussion in the previous paragraph, the proposed RRH control involves a smaller number of RRHs in cooperative transmission with increase of $P$ due to the saturation characteristic of the log function. Accordingly, the proposed RRH control further reduces RS overhead according to the increase of $P$, achieving remarkable network capacity improvement. For example, the proposed RRH control with greedy search-based and LP relaxation-based algorithms improves network capacity by 33 and $20 \%$ over one nearest RRH selection when transmission power per spatial stream becomes $30 \mathrm{dBm}$.

\section{Conclusions}

In this paper, we have developed an RRH control scheme for FDD-based C-RANs which maximizes the network capacity by adaptively determining a cooperating RRH subset in consideration of both the antenna processing gain and RS overhead. Our main contribution is introducing a realistic performance metric that takes into account both antenna processing gain and RS transmission overhead, which then leads to formulation of a network capacity maximization problem. This has never been studied in the literature. Simulation results have revealed that larger number of antennas do not always guarantee network capacity increase in FDD-based C-RANs. The proposed algorithm adaptively finds the best RRH subset for cooperation and offers a significant improvement on the network capacity. For future research directions, the proposed RRH control may be extended to incorporate transmit power control and uplink CSI overhead.

\section{Endnotes}

${ }^{1}$ Later in Section 4, we will validate that Jensen's inequality provides close approximation for the long-term ergodic network capacity in a C-RAN.

${ }^{2}$ We consider CSI-RS transmission overhead in commercial LTE systems. CSI-RS configurations with 5-ms period result in $0.12 \%$ of time and frequency resource consumption per antenna [25].

\section{Competing interests}

The authors declare that they have no competing interests.

\section{Acknowledgments}

This work was supported by supported by the National Research Foundation of Korea (NRF) grant funded by the Korea government (MSIP) (No. 2014R1A2A2A01003637), and Basic Science Research Program through the National Research Foundation of Korea (NRF) funded by the Ministry of Education (No. NRF-2015R1D1A1A01057100).

\section{Author details}

${ }^{1}$ Department of Electrical and Computer Engineering, INMC, Seoul National University, Gwanak-ro, 08826 Seoul, South Korea. ${ }^{2}$ Department of Electronics Engineering, Gachon University, Seongnamdae-ro, 13120, Seongnam, South Korea.
Received: 2 December 2015 Accepted: 9 June 2016

Published online: 22 July 2016

\section{References}

1. P Demestichas, A Georgakopoulos, D Karvounas, KTsagkaris, V Stavroulaki, J Lu, C Xiong, J Yao, 5 G on the horizon: key challenges for the radio-access network. IEEE Veh. Technol. Mag. 8(3), 47-53 (2013)

2. P Rost, CJ Bernardos, AD Domenico, MD Girolamo, M Lalam, A Maeder, D Sabella, D Wubben, Cloud technologies for flexible $5 \mathrm{G}$ radio access networks. IEEE Commun. Mag. 52(5), 68-76 (2014)

3. J Wu, Z Zhang, Y Hong, Y Wen, Cloud radio access network (C-RAN): a primer. IEEE Netw. 29(1), 35-41 (2015)

4. M Peng, Y Li, Z Zhao, C Wang, System architecture and key technologies for $5 \mathrm{G}$ heterogeneous cloud radio access networks. IEEE Network. 29(2), 6-14 (2015)

5. China Mobile. C-RAN: the road towards green RAN, (2011). White Paper

6. Ericsson. Heterogeneous Networks: meeting mobile broadband expectations with maximum efficiency, (2012). White Paper

7. SK Telecom. SK Telecom's view on $5 \mathrm{G}$ vision, architecture, technology, and spectrum, (2014). White Paper

8. S Park, O Simeone, O Sahin, S Shamai, Robust and efficient distributed compression for cloud radio access networks. IEEE Trans. Veh. Technol. 62(2), 692-703 (2013)

9. S Park, O Simeone, O Sahin, S Shamai, Joint precoding and multivariate backhaul compression for the downlink of cloud radio access networks. IEEE Trans. Signal Process. 61(22), 5646-5658 (2013)

10. S Park, O Simeone, O Sahin, S Shamai, Joint decompression and decoding for cloud radio access networks. IEEE Signal Process. Lett. 20(5), 503-506 (2013)

11. L Zhou, W Yu, Uplink multicell processing with limited backhaul via per-base-station successive interference cancellation. IEEE J. Sel. Areas Commun. 30(10), 1981-1993 (2013)

12. Y Zhou, W Yu, Optimized backhaul compression for uplink cloud radio access network. IEEE J. Sel. Areas Commun. 32(6), 1295-1307 (2014)

13. J Kang, O Simeone, J Kang, S Shamai, Joint signal and channel state information compression for the backhaul of uplink network MIMO systems. IEEE Trans. Wireless Commun. 13(3), 1555-1567 (2014)

14. B Dai, W Yu, Sparse beamforming and user centric clustering for downlink cloud radio access network. IEEE Access. 2, 1326-1339 (2014)

15. H Zhang, $H$ Liu, C Jiang, X Chu, A Nallanathan, X Wen, A practical semi-dynamic clustering scheme using affinity propagation in cooperative picocells. IEEE Trans. Veh. Technol. 99, 1-6 (2014)

16. A Liu, VKN Lau, Joint power and antenna selection optimization in large cloud radio access networks. IEEE Trans. Signal Process. 62(5), 1319-1328 (2014)

17. Y Shi, J Zhang, KB Letaief, in IEEE International Conference on Communications. CSI overhead reduction with stochastic beamforming for cloud radio access networks, (Sydney, 2014)

18. B Hassibi, BM Hochwald, How much training is needed in multiple-antenna wireless links? IEEE Trans. Inform. Theory. 49(4), 951-963 (2003)

19. X Ma, L Yang, GB Giannakis, Optimal training for MIMO frequency-selective fading channels. IEEE Trans. Wireless Commun. 4(2), 453-466 (2005)

20. 3rd Generation Partnership Project, LTE, Evolved Universal Terrestrial Radio Access (EUTRA); Physical Channels and Modulation. 3GPP TS 36.211 version 10.7.0 Release 10 (2013)

21. A Dua, K Medepalli, AJ Paulraj, Receive antenna selection in MIMO systems using convex optimization. IEEE Trans. Wireless Commun. 5(9), 2353-2357 (2006)

22. A Snyman, Practical Mathematical Optimization: an Introduction to Basic Optimization Theory and Classical and New Gradient-Based Algorithms. (Springer, Spring Street, New York, NY 10013, USA, 2005)

23. M Peng, S Yan, HV Poor, Ergodic capacity analysis of remote radio head associations in cloud radio access networks. IEEE Wireless Commun. Lett. 3(4), 365-368 (2014)

24. LG Electronics, R1-111628, Phase 1 CoMP simulation evaluation results and analysis for full buffer. 3GPP TSG RAN WG1 (2011)

25. S Ahmadi, A Practical Systems Approach to Understanding 3GPP LTE Release 10 and 11 Radio Access Technologies. (Academic Press, 225 Wyman Street, Waltham, MA 02451, USA, 2013) 